\title{
A new and homogeneous metallicity scale for Galactic classical Cepheids
}

\section{Physical parameters $\star, \star \star, \star \star \star$}

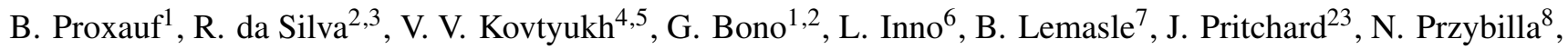 \\ J. Storm ${ }^{9}$, M. A. Urbaneja ${ }^{8}$, E. Valenti ${ }^{23}$, M. Bergemann ${ }^{6}$, R. Buonanno ${ }^{1,10}$, V. D’Orazi ${ }^{11,12}$, M. Fabrizio ${ }^{2,3}$, \\ I. Ferraro ${ }^{2}$, G. Fiorentino ${ }^{13}$, P. François ${ }^{14,15}$, G. Iannicola ${ }^{2}$, C. D. Laney ${ }^{16,17}$, R.-P. Kudritzki ${ }^{18,19,20}$, N. Matsunaga ${ }^{21}$, \\ M. Nonino ${ }^{22}$, F. Primas ${ }^{23}$, M. Romaniello ${ }^{23}$, and F. Thévenin ${ }^{24}$
}

(Affiliations can be found after the references)

Received 23 March 2018 / Accepted 30 April 2018

\begin{abstract}
We gathered more than 1130 high-resolution optical spectra for more than 250 Galactic classical Cepheids. The spectra were collected with the optical spectrographs UVES at VLT, HARPS at $3.6 \mathrm{~m}$, FEROS at $2.2 \mathrm{~m} \mathrm{MPG/ESO,} \mathrm{and} \mathrm{STELLA.} \mathrm{To} \mathrm{improve} \mathrm{the} \mathrm{effective} \mathrm{temperature}$ estimates, we present more than 150 new line depth ratio (LDR) calibrations that together with similar calibrations already available in the literature allowed us to cover a broad range in wavelength $(5348 \leq \lambda \leq 8427 \AA)$ and in effective temperature $\left(3500 \leq T_{\text {eff }} \leq 7700 \mathrm{~K}\right)$. This gives us the unique opportunity to cover both the hottest and coolest phases along the Cepheid pulsation cycle and to limit the intrinsic error on individual measurements at the level of $\sim 100 \mathrm{~K}$. As a consequence of the high signal-to-noise ratio of individual spectra, we identified and measured hundreds of neutral and ionized lines of heavy elements, and in turn, have the opportunity to trace the variation of both surface gravity and microturbulent velocity along the pulsation cycle. The accuracy of the physical parameters and the number of $\mathrm{Fe}_{\mathrm{I}}$ (more than one hundred) and Fe II (more than ten) lines measured allowed us to estimate mean iron abundances with a precision better than 0.1 dex. We focus on 14 calibrating Cepheids for which the current spectra cover either the entire or a significant portion of the pulsation cycle. The current estimates of the variation of the physical parameters along the pulsation cycle and of the iron abundances agree very well with similar estimates available in the literature. Independent homogeneous estimates of both physical parameters and metal abundances based on different approaches that can constrain possible systematics are highly encouraged.
\end{abstract}

Key words. Galaxy: disk - stars: abundances - stars: fundamental parameters - stars: variables: Cepheids - stars: oscillations

\section{Introduction}

Radially variable stars played a crucial role in the transition from qualitative to quantitative astrophysics. The reasons are manifold. They are simultaneously excellent primary distance indicators and very robust stellar tracers. The most popular of these stars are ( $i$ ) RR Lyrae: old ( $t>10 \mathrm{Gyr}$ ), low-mass stars; (ii) Mira: intermediate-age (from a few hundred Myr to several Gyr) stars; and (iii) classical Cepheids: young (from tens of Myr to a few hundred Myr) stars. Pulsation and evolutionary observables have been adopted for more than one century to constrain Galactic stellar populations (Baade 1958) and, in particular, to improve our knowledge of the physical mechanisms driving their pulsation properties and evolution (Kraft 1957; Preston 1964; Preston et al. 1965; Wallerstein 1972, 1979).

* Partly based on observations made with ESO Telescopes at the La Silla/Paranal Observatories under program IDs: 072.D-0419, 073.D0136 and 190.D-0237 for HARPS spectra; 084.B-0029, 087.A-9013, 074.D-0008, 075.D-0676 and 60.A-9120 for FEROS spectra; 081.D0928, 082.D-0901, 089.D-0767 and 093.D-0816 for UVES spectra.

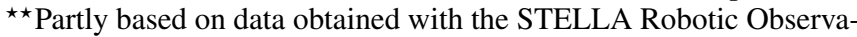
tory in Tenerife, an AIP facility jointly operated by AIP and IAC.

$\star \star \star$ Tables 3-5 are only available at the CDS via anonymous ftp to cdsarc.u-strasbg.fr $(130.79 .128 .5)$ or via http://cdsarc.u-strasbg.fr/viz-bin/qcat?J/A+A/616/A82
In this context, classical Cepheids (CCs) have been the crossroad of a paramount theoretical effort (Bono et al. 1999a,b; Fiorentino et al. 2007; Marconi et al. 2005; Anderson et al. 2016) and observational (Riess et al. 2016; Freedman \& Madore 2010; Gieren et al. 2013; Pietrzyński et al. 2013; Soszyński et al. 2017). These stars are the most popular primary distance indicators used to calibrate secondary indicators and to estimate the Hubble constant. Classical Cepheids are bright $\left(-2 \leq M_{\mathrm{V}} \leq-7\right.$ mag $)$ and recent photometric investigations based on ground-based and/or space facilities provide accurate mean magnitudes for Cepheids located in external galaxies in the Local Group and in the Local Volume (Bono et al. 2010; Macri et al. 2015; Hoffmann et al. 2016, and references therein).

However, the spectroscopic investigations are lagging, and indeed they have been mainly focused on Galactic Cepheids (Luck et al. 2011; Luck \& Lambert 2011; Wallerstein et al. 2015; Genovali et al. 2014, 2015; Lemasle et al. 2013; da Silva et al. 2016, and references therein) and on a few nearby stellar systems such as the Magellanic Clouds (Luck et al. 1998, Romaniello et al. 2008; Lemasle et al. 2017). Classical Cepheids are also excellent physics laboratories, and indeed they have been used to investigate their dynamical properties along the pulsation cycle. They have been investigated both in the optical (Struve 1944; Kraft 1956) and near-infrared (NIR) regime (Sasselov et al. 1989; Sasselov \& Lester 1990b). More recently, they have also been studied by Nardetto et al. (2009) to constrain 
the variation of the projection factor in a very exhaustive paper by Wallerstein et al. (2015) and in the validation of the quasistatic approximation by Vasilyev et al. $(2017,2018)$.

The elemental abundances are in a positive status since we are approaching an almost complete spectroscopic census of the currently known Galactic Cepheids ( 450) based on high-resolution and high signal-to-noise $(\mathrm{S} / \mathrm{N})$ optical spectra (Bono et al., in prep.). Our group has been involved in a longterm project (DYONISOS) aimed at providing a homogeneous metallicity scale for field and cluster Galactic and Magellanic Cepheids. The current analyses mainly rely on the classical quasi-static approximation, in which the spectra of a CC, randomly collected along the pulsation cycle, are approximated with the physical properties of a static star with similar effective temperature, surface gravity, and microturbulent velocity.

One of the key problems in dealing with spectroscopy of variable stars in the Cepheid instability strip is that the effective temperature, when moving from minimum to maximum light, changes by roughly $1000 \mathrm{~K}$. At the same time, the surface gravity also changes by up to $0.8-0.9$ dex. These variations are correlated with the luminosity amplitude. The quasi-static approximation becomes more severe in dealing with spectra collected across pulsation phases affected by nonlinear phenomena (formation and propagation of shocks), i.e., the phases along the rising branch or just before maximum compression. Bono et al. (2000b) provide a detailed discussion concerning these phenomena and their interplay with the Hertzsprung progression.

The effective temperature of CCs can be estimated using color-temperature relations, but this approach requires very accurate optical and NIR photometry. Moreover, this approach is prone to possible systematics introduced by reddening uncertainties and/or metallicity dependence. A very promising independent approach has been recently provided by Kervella et al. (2004) and Mérand et al. (2015) using optical and NIR interferometric measurements of the diameter of nearby CCs. The same applies to the infrared surface brightness (IRSB) method by Storm et al. (2011a, b) and by Groenewegen (2008) using optical and NIR photometry and radial velocities to constrain the angular diameter variations. In this context, a temperature diagnostic that appears very robust is the line depth ratio (LDR). It relies on plain physical assumptions: the depth ratio of several pairs of absorption lines is strongly correlated with the effective temperature. To minimize the dependence of the abundance on the surface gravity and possible uncertainties in the continuum location, the lines forming these pairs should come from the same (or a similar) element, have similar wavelengths, be weak, nonsaturated, and come from neutral species (Gray 2005).

The use of the LDR method to estimate the effective temperature of CCs was pioneered by several authors, i.e., Sasselov \& Lester (1990a), Krockenberger et al. (1998), Kovtyukh \& Gorlova (2000; hereinafter KG00), and by Kovtyukh et al. (2003b; hereinafter K03). In particular, KG00 provided a set of calibrations based on 32 pairs of lines. The K03 work provided 105 new LDR calibrations using more than 180 FGK main sequence stars covering $\sim 1$ dex in iron abundance $(-0.5 \leq[\mathrm{Fe} / \mathrm{H}] \leq 0.5)$ and for which high-resolution $(R \sim 42000)$, high $\mathrm{S} / \mathrm{N}$ spectra were available together with accurate trigonometric parallaxes from HIPPARCOS and effective temperature estimates with an accuracy on the order of $1 \%$. Subsequently, Kovtyukh (2007; hereinafter K07) obtained a set of 131 LDR calibrations using 161 FGK supergiants, increasing the valid range in temperature to about 3600-7800 K (F0 I$\mathrm{K} 5 \mathrm{I})$. The equivalent width (EW) measurements of each pair provide an independent estimate of the effective temperature.
These LDRs have been very successful and have been used in many recent spectroscopic investigations of CCs (e.g., Andrievsky et al. 2002a, b; Lemasle et al. 2007, 2008; Genovali et al. 2013, 2014) with a typical precision on the order of $150 \mathrm{~K}$.

The main aim of this investigation is to provide new and homogeneous estimates of the intrinsic parameters (effective temperature, surface gravity, and microturbulent velocity) for $\mathrm{CCs}$ and to constrain their impact on the iron abundance. The key advantage of the current investigation when compared with similar analyses available in the literature is that we are dealing with 14 calibrating CCs covering a broad range in pulsation period $(0.53 \leq \log P \leq 1.62)$ and in metal abundance $(-0.11 \leq[\mathrm{Fe} / \mathrm{H}] \leq 0.35 \mathrm{dex})$. The current sample was defined as calibrating CCs, since the optical high-resolution spectra cover the pulsation cycle either fully or for the most part. Indeed, the number of spectra per object range from five (XX Sgr) to more than one hundred $(\zeta$ Gem).

The structure of the paper is the following. In Sect. 2 we present the entire spectroscopic dataset and we discuss the $\mathrm{S} / \mathrm{N}$ and wavelength range covered by the spectra. In Sect. 3 we discuss the strategies adopted to pre-reduce and calibrate the spectra. Section 3 deals with the adopted line lists and the approach adopted to measure the EWs. In Sect. 4 we discuss the determination of the atmospheric parameters and radial velocities, including the LDR calibrations used to derive the effective temperature and their validity range. The determination of the iron abundances is presented in Sect. 5, and the variations along the pulsation cycle are discussed in Sect. 6. The summary of the results and future perspective of this project are given in Sect. 7.

\section{Spectroscopic datasets}

The spectroscopic datasets partly analyzed in the current paper, and that will be used for a new series of papers, are spectra collected at three different telescopes of the European Southern Observatory (ESO): the Ultraviolet and Visual Echelle Spectrograph (UVES; Dekker et al. 2000) at the Very Large Telescope, the High Accuracy Radial velocity Planet Searcher (HARPS; Mayor et al. 2003) at the $3.6 \mathrm{~m}$, and the Fiber-fed Extended Range Optical Spectrograph (FEROS; Kaufer et al. 1999) at the $2.2 \mathrm{~m} \mathrm{MPG/ESO}$. A list of CCs was defined for the three spectrographs and the related spectra were downloaded from the ESO archive, forming the datasets UVES TS (for this study, 32 spectra, 3 targets), HARPS (199 spectra, 9 targets), and FEROS TS (486 spectra, 169 targets).

The quoted spectra were complemented with spectra collected by Inno et al. (ID: 093.D-0816, dataset UVES IN, 154 spectra, 46 targets), Kovtyukh et al. (2016, dataset UVES KO, 9 spectra, 1 target, and dataset FEROS KO, 2 spectra, 2 targets), Genovali et al. (2015, dataset UVES GE, 120 spectra, 73 targets). We also included 134 high-resolution spectra for 5 targets collected with the STELLA Echelle Spectrograph (SES; Strassmeier et al. 2004, 2010). As a whole, we ended up with 1136 high-resolution spectra for 251 Cepheids, explicitly accounting for the multiplicity of objects among the different spectrographs.

The spectral resolution of the quoted spectrographs for the instrument settings used are $R \sim 40000$ (UVES), $R \sim 115000$ (HARPS), $R \sim 48000$ (FEROS), and $R \sim 55000$ (STELLA). The corresponding wavelength ranges for our sample are (i) UVES: 3050-3870 ̊; 3760-4985 А, 5684-7520

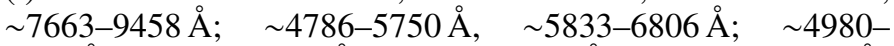
$5952 \AA, \quad \sim 6035-7002 \AA ; \quad \sim 6700-8523 \AA, \quad \sim 8659-10422 \AA$; 
(ii) HARPS: 3781-5304,$~ 25337-6912 \AA$; (iii) FEROS: 4000-9216 ̊; and (iv) STELLA: 3872-8813. .

The current spectroscopic dataset can be divided into three different subgroups:

(1) Calibrating Cepheids:

(a) Phase dependence - For 14 targets in our sample the spectra cover either a significant part or the entire pulsation cycle. This is the subsample of CCs that we analyze in the present paper and that we adopt to constrain the accuracy of the intrinsic parameters and, in particular, their impact on the iron abundance. We note that the bulk of CCs are strictly periodic on long evolutionary timescales. This means that we take advantage of the strict periodicity in cyclic variations.

(b) Cluster Cepheids - Our sample includes 14 CCs that are candidate cluster variables. The number of spectra per target ranges from 1 to more than 100. These targets will be adopted to link field and cluster CCs on the same metallicity scale.

(2) Cepheids with new iron abundances: This subgroup includes roughly 50 Cepheids for which we secured high-resolution UVES spectra and for which no metallicity estimate is available in the literature. The number of spectra per target ranges from 1 to more than 50 .

(3) Cepheids with homogeneous iron abundances: We derive homogeneous iron abundances based on high-resolution spectra for 216 CCs. The number of spectra per target ranges from 1 to almost 50 .

In the current investigation we focus on the 14 calibrating CCs with multi-epoch spectroscopic measurements. Details on the number of spectra are given in Table 1. The spectra of EV Sct and X Sgr were initially included in our analysis, but excluded afterward. In a detailed spectroscopic investigation based on high-resolution spectra, Kovtyukh \& Andrievsky (1999) found that EV Sct shows strong line asymmetries and even split lines. They suggested that this object might be a binary system with two short period Cepheids in spite of the observed phase coherence. Concerning X Sgr, Mathias et al. (2006), on the basis of both optical and NIR high-resolution spectroscopy, found strong dynamical variations in the outermost layers. Moreover, optical and NIR interferometric data (Li Causi et al. 2013; Gallenne et al. 2014) showed evidence of a possible companion, which is also suggested by the orbital velocity curve determined by Feast et al. (2008). Szabados (1990, 2003), Evans (1992), and Groenewegen (2008) provide a more detailed discussion. These two CCs were also included in the list of candidate nonradial pulsators by Kovtyukh et al. (2003a).

\section{Data reduction and analysis}

The spectra have to be prepared for the later analysis by doing an initial pre-reduction (up to the wavelength calibration step). The spectra from UVES and HARPS (Phase 3) were already prereduced by their own pipeline. The FEROS spectra were reduced with a modified version of the FEROS Data Reduction System (DRS) pipeline developed by one of us (J. Pritchard). Several FEROS spectra taken before 2004 could not be reduced because of a change in the detector software architecture from the Brorfelde Image Acquisition System (BIAS) controller to the Fast Imager Electronic Readout Assemby (FIERA) and therefore a different file structure. The current FEROS sample includes 355 spectra; the remaining 133 are not yet included in the analysis.
The next step, the continuum normalization, was required for UVES (except UVES GE, already continuum normalized), HARPS, and FEROS. Before the normalization, UVES and HARPS spectra were split into blue and red spectral parts owing to the big central gap present therein. The continuum normalization was performed using the Image Reduction and Analysis Facility (IRAF ${ }^{1}$ ) by fitting cubic spline functions ' to a set of continuum windows visually selected in the spectra. For UVES and HARPS spectra we normally used first order functions, but for FEROS spectra high-order (20-50) cubic spline functions were required given their large wavelength range.

The radial velocity of the objects was determined using IRAF by cross-correlating the target spectrum with an observed solar template spectrum in the rest frame (Solar Flux Atlas; Kurucz et al. 1984) degraded to the UVES resolution. The UVES GE spectra were already in the rest frame and served as templates. For FEROS spectra, because of possible contamination due to bleeding during the wavelength calibration (François et al. 2006), we preferred to adopt the radial velocities derived by the routine used to measure the EWs (see Sect. 3). For STELLA spectra, the radial velocities come directly from the STELLA reduction pipeline (Weber et al. 2012), which is based on IRAF. It performs the standard data reduction steps including scattered light removal and continuum normalization. The radial velocities are based on cross-correlation with a synthetic template spectrum. In the case of Cepheids a G-type star template was used. The resulting radial velocities have estimated uncertainties of about $0.2 \mathrm{~km} \mathrm{~s}^{-1}$.

The spectra were examined for EW measurements by checking the $\mathrm{S} / \mathrm{N}$ in various continuum regions in the spectra. From the preliminary $\mathrm{S} / \mathrm{N}$ estimates derived from these blocks in combination with a visual check, the spectra were classified as low, intermediate, or high-quality exposures, and the usability for further analysis, especially the metallicity determination, was evaluated. The UVES GE sample has already been marked with sufficient $\mathrm{S} / \mathrm{N}$ by the original authors. The UVES KO and UVES IN S/Ns could be taken directly from the ESO archive. For FEROS the S/Ns range from 25 to 475 , for HARPS they are between 145 and 400, and for UVES TS they cover a range between 235 and 480. We noticed that many of the FEROS spectra have been classified as low-quality exposures, and have thus not been included in the metallicity and effective temperature determination. Examples of HARPS, UVES, FEROS, and STELLA spectra of different metallicities and with different $\mathrm{S} / \mathrm{N}$ estimates (measured around $6000 \AA$ ) are depicted in Fig. 1 .

The EWs were measured using the Automatic Routine for line Equivalent widths in stellar Spectra (ARES; Sousa et al. 2007, 2015). First, a global set of common input parameters was used, and then the parameters were individually adjusted, giving better fits of the spectral line profiles. As mentioned in Sect. 3, ARES also performs an automatic estimate of the radial velocity, whose values were used in the case of FEROS spectra.

Three line lists were created:

(a) one built by combining four individual line lists received from Kovtyukh and used to derive the effective temperature ( $\left.T_{\text {eff }}\right)$ of the objects (153 lines);

(b) one from Genovali et al. (2013), complemented with the Gaia-ESO Survey (GES, Gilmore et al. 2012; Randich et al. 2013), and cross-checked with the Vienna Atomic Line Database (VALD3, Ryabchikova et al. 2015), containing iron features (615 lines);

1 Distributed by the National Optical Astronomy Observatories (NOAO), USA. 
Table 1. Calibrating Cepheids for which high-resolution spectra cover either a substantial fraction or the entire pulsation cycle.

\begin{tabular}{lccccccccccc}
\hline \hline Name & $R_{\mathrm{G}}^{a} \pm \sigma(\mathrm{pc})$ & $\alpha_{\mathrm{ICRS}}$ & $\delta_{\text {ICRS }}$ & Period $^{b}($ days) & $T_{0}^{b}-2400000$ (days) & {$[\mathrm{Fe} / \mathrm{H}]_{\text {lit }}^{a} \pm \sigma$} & $N_{\mathrm{F}}$ & $N_{\mathrm{H}}$ & $N_{\mathrm{U}}$ & $N_{\mathrm{S}}$ & $N_{\text {tot }}$ \\
\hline V340 Ara & $4657 \pm 427$ & $16: 45: 19.112$ & $-51: 20: 33.393$ & 20.80876 & 44881.2740 & $0.33 \pm 0.09$ & 26 & $\ldots$ & 6 & $\ldots$ & 32 \\
$\eta$ Aql & $7750 \pm 452$ & $19: 52: 28.368$ & $+01: 00: 20.370$ & 7.17679 & 43368.8611 & $0.14 \pm 0.02$ & $\ldots$ & $\ldots$ & $\ldots$ & 11 & 11 \\
S Cru & $7593 \pm 451$ & $12: 54: 21.998$ & $-58: 25: 50.214$ & 4.68973 & 44301.5560 & $0.08 \pm 0.10$ & 1 & 12 & $\ldots$ & $\ldots$ & 13 \\
$\beta$ Dor & $7936 \pm 451$ & $05: 33: 37.517$ & $-62: 29: 23.369$ & 9.84308 & 47913.0970 & $-0.06 \pm 0.10$ & $\ldots$ & 46 & $\ldots$ & $\ldots$ & 46 \\
$\zeta$ Gem & $8273 \pm 452$ & $07: 04: 06.531$ & $+20: 34: 13.074$ & 10.15072 & 50139.4010 & $-0.11 \pm 0.10$ & $\ldots$ & 47 & $\ldots$ & 81 & 128 \\
Y Oph & $7141 \pm 452$ & $17: 52: 38.702$ & $-06: 08: 36.870$ & 17.12415 & 44083.4490 & $0.12 \pm 0.04$ & $\ldots$ & 8 & $\ldots$ & $\ldots$ & 8 \\
RS Pup & $8585 \pm 444$ & $08: 13: 04.216$ & $-34: 34: 42.696$ & 41.44002 & 53014.2808 & $0.21 \pm 0.10$ & $\ldots$ & 15 & $\ldots$ & $\ldots$ & 15 \\
UZ Sct & $5309 \pm 448$ & $18: 31: 22.368$ & $-12: 55: 43.350$ & 14.7482 & 45496.3631 & $0.33 \pm 0.08$ & 28 & $\ldots$ & 6 & $\ldots$ & 34 \\
AV Sgr & $5980 \pm 454$ & $18: 04: 48.780$ & $-22: 43: 56.600$ & 15.41153 & 53109.1989 & $0.35 \pm 0.17$ & 28 & $\ldots$ & 5 & $\ldots$ & 33 \\
VY Sgr & $5862 \pm 453$ & $18: 12: 04.568$ & $-20: 42: 14.580$ & 13.55845 & 50891.6007 & $0.33 \pm 0.12$ & 30 & $\ldots$ & 4 & $\ldots$ & 34 \\
XX Sgr & $6706 \pm 453$ & $18: 24: 44.501$ & $-16: 47: 49.816$ & 6.42414 & 44822.6740 & $-0.01 \pm 0.06$ & $\ldots$ & $\ldots$ & 5 & $\ldots$ & 5 \\
Y Sgr & $7483 \pm 452$ & $18: 21: 22.986$ & $-18: 51: 36.002$ & 5.77335 & 40762.4310 & $0.11 \pm 0.03$ & $\ldots$ & 20 & $\ldots$ & 3 & 23 \\
R TrA & $7519 \pm 451$ & $15: 19: 45.713$ & $-66: 29: 45.742$ & 3.38924 & 52365.1127 & $0.16 \pm 0.11$ & 1 & 14 & $\ldots$ & $\ldots$ & 15 \\
RZ Vel & $8249 \pm 445$ & $08: 37: 01.303$ & $-44: 06: 52.848$ & 20.39689 & 45003.4620 & $0.19 \pm 0.10$ & 1 & 11 & $\ldots$ & $\ldots$ & 12 \\
\hline
\end{tabular}

Notes. From left to right the columns give the name, galactocentric distance, right ascension, declination, pulsation period, and zero-phase reference epoch of maximum light in the $V$ band. Column 7 lists the iron abundance available in the literature. Columns 8-11 show the number of optical spectra used for each spectrograph: $N_{\mathrm{F}}$, FEROS; $N_{\mathrm{H}}$, HARPS; $N_{\mathrm{U}}$, UVES; $N_{\mathrm{S}}$, STELLA. The last column lists the total number of spectra per target.

References. ${ }^{(a)}$ Genovali et al. (2014); ${ }^{(b)}$ this investigation.

(c) one from Genovali et al. (2015) and da Silva et al. (2016) including lines belonging to other elements (113 lines, $\alpha$, s, and $\mathrm{r}$ elements).

The atomic lines used for effective temperature determination are listed in Table 3. The line list of the other elements will be discussed in a forthcoming paper.

\section{Atmospheric parameters}

\subsection{New and old LDR calibrations}

Although being widely used, the LDRs by KG00 are based on polynomial relations hampered by a limited range in effective temperature (4700-6700 K) and in wavelength (5670-6850 A). The former limitation affects the accuracy when dealing with spectra collected across the hottest pulsation phases; the latter affects the accuracy when dealing with spectra that have higher $\mathrm{S} / \mathrm{N}$ in redder wavelengths $(\lambda>6500 \AA)$. For this reason, K07 extended the number of calibrations to a total of 131 pairs of lines located in the wavelength range between 5348 and $6768 \AA$. The key advantage of the new LDRs is that they allow effective temperature estimates up to $7800 \mathrm{~K}$.

To further improve the range in wavelength covered by the LDR calibrations, one of us (V. Kovtyukh) identified 151 new pairs of lines. Here we publish these new calibrations, which are based on effective temperatures estimated by K07. These authors fixed the temperature scale using nonvariable supergiants for which the effective temperature was already available in the literature using independent approaches. Subsequently, they applied the new temperature scale to classical Cepheids and they only retained the LDRs for which the standard deviation was smaller than $110 \mathrm{~K}$.

In case the same pair appears in different calibrations, we always selected the most recent. All in all we ended up with 257 pairs of lines ranging from 5348 to $8427 \AA$ and covering the temperature range from 3500 to $7700 \mathrm{~K}$. To our knowledge this is the most complete list of LDRs ever compiled for F-K spectral type stars. Moreover, as shown in Fig. 2, the standard deviations of the effective temperature determinations are clearly reduced with the increasing number of calibrations. The figure shows the results for the HARPS sample, but it is also valid for the other samples.

We are dealing therefore with three different sets of LDR calibrations:

(a) KG00: 32 calibrations;

(b) K07: 131 calibrations (the analytical functions are shown in the present work for the first time);

(c) K18 (this investigation): 151 new calibrations.

From the 257 calibrations (multiplicity removed), based on 153 lines, 257 independent estimates of the effective temperature could be obtained. Table 3 contains an excerpt of the list of the analytical relations for the LDRs used in this investigation. The complete table is available in electronic form at the CDS.

\subsection{Effective temperature estimation}

Several calibrations were removed since they provide effective temperatures that are significantly different from the bulk of the LDRs. Such outliers in the temperature distribution may be caused either by blends in the specific pair or by limited $\mathrm{S} / \mathrm{N}$. Each calibration also has a certain range of validity where the LDR can be used. For many of our sample stars we did not have a previous estimate of their effective temperature, therefore, we performed an iterative process. In each iteration, the calibrations had to pass a sigma clipping and were then accepted only if a defined interval around the median temperature was overlapping with the temperature range of the calibration. The clippings were stopped when no more calibrations were cut.

Once the clippings were finished, the mean and median temperature was computed from the remaining values. The number of calibrations that survived the sigma clipping was typically high $(\sim 100)$ such that a solid statistical basis was present and the standard deviations were relatively low $(<100 \mathrm{~K})$ for most of the spectra.

We performed a number of numerical simulations assuming no sigma clipping and we found that the mean/median values are minimally affected, while the standard deviation increases to $\sim 150 \mathrm{~K}$. The approach we adopted to estimate the effective 
B. Proxauf et al.: A new and homogeneous metallicity scale for Galactic classical Cepheids. I.

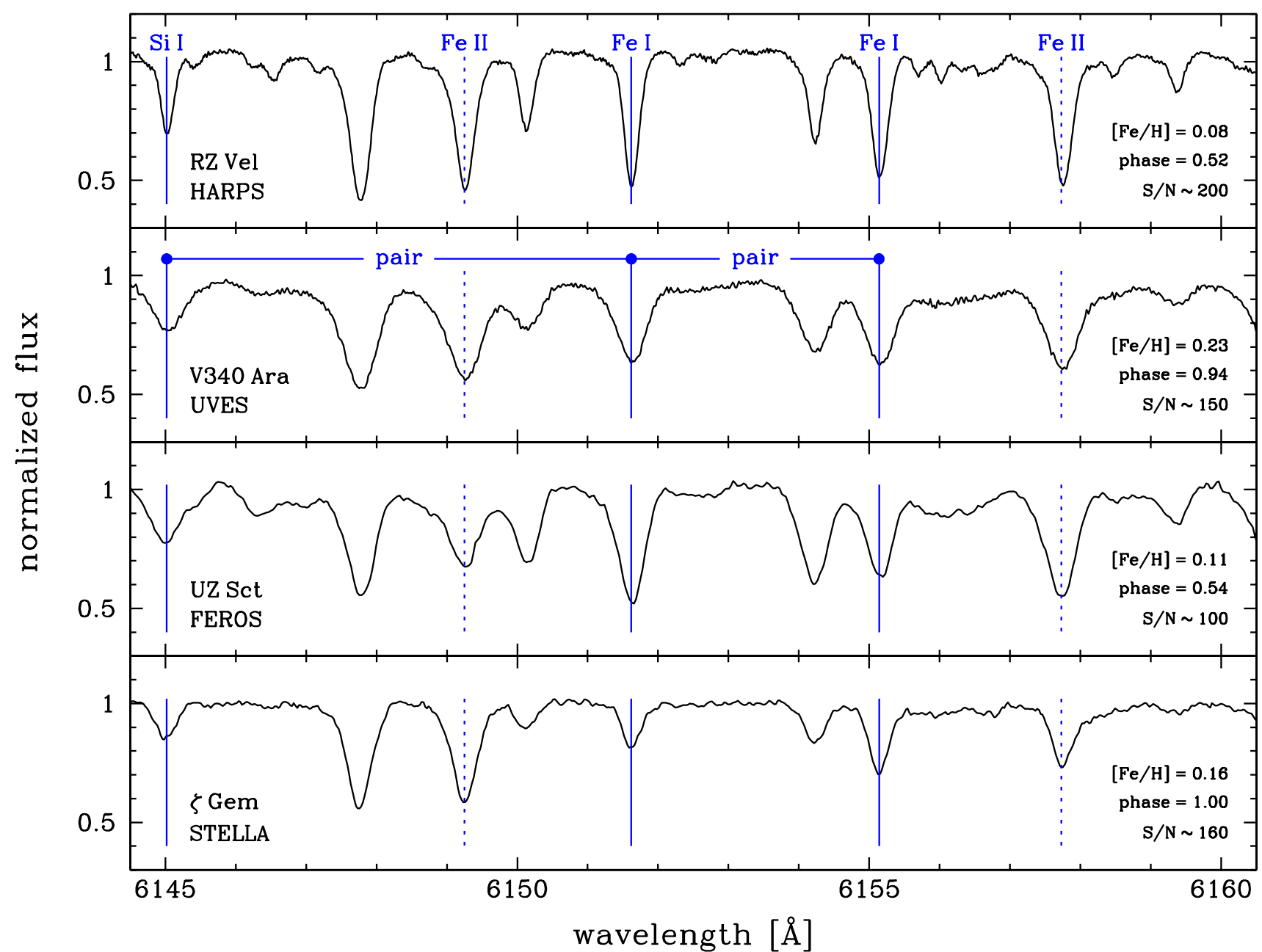

Fig. 1. Examples of high-resolution spectra for various calibrating classical Cepheids collected with the spectrographs HARPS, UVES, FEROS, and STELLA. The vertical lines indicate the position either of iron lines only used for the metallicity determination (dashed) or of lines also adopted in the LDR method (solid). The name of the Cepheid, its mean metallicity, phase, and S/N of these example spectra around $6000 \AA$ are also labeled.

temperature for the individual spectra can be summarized as follows:

(a) Take all available LDR calibrations and calculate individual effective temperatures.

(b) Calculate the effective temperature mean $/$ median $/ \sigma$.

(c) If the effective temperature of a given LDR calibration is out of $2 \sigma$ from the median and $\sigma>100 \mathrm{~K}$, discard the LDR; if no LDR calibration is discarded go to $(\mathrm{d})$, otherwise go back to $(\mathrm{b})$.

(d) Check if the median value is within the validity range of the calibrations; discard all the LDR calibrations that do not cover the derived median; if no LDR calibration is discarded go to (e), otherwise go back to (b).

(e) Calculate the final mean $/$ median $/ \sigma$.

Table 4 contains an excerpt of the median values of effective temperatures and standard deviations, together with other parameters, derived for individual spectra of the calibrating Cepheids. Table 5 contains an excerpt of the list of spectra for which the effective temperature could not be estimated, as explained in Sect. 3. The complete tables are available in electronic form at the CDS.

In order to validate the effective temperature estimates based on the new sets of LDRs, we performed a detailed comparison with similar estimates available in the literature. The top panel of Fig. 3 shows the comparison between the current effective temperature amplitude $\left(\Delta T_{\mathrm{eff}}=T_{\mathrm{eff}}{ }^{\max }-T_{\mathrm{eff}}{ }^{\mathrm{min}}\right)$ and similar estimates for 60 Galactic Cepheids for which Storm et al. (2011a; hereinafter S11) applied the IRSB method to estimate individual distances and intrinsic parameters. We estimated the $\Delta T_{\text {eff }}$ by fitting the effective temperature curves with sinusoidal functions. Cepheids for which the phase coverage is not optimal (Y Oph, UZ Sct, AV Sgr, and XX Sgr) are indicated with black crosses. The vertical error bars show the standard deviations on the fitted functions. Typical error bars on the $\Delta T_{\text {eff }}$ values from $\mathrm{S} 11$ are smaller than the symbol size. Data plotted in this panel show the typical V-shape distribution (Bono et al. 2000a), i.e., pulsation amplitudes display a well-defined minimum across the so-called Hertzsprung progression $(\log P \sim 1.0$, Bono et al. 2000b). The agreement between the two data sets is very good in the period range in common.

We also compared the current $\Delta T_{\text {eff }}$ estimates with similar evaluations provided by Pel (1978) using multiband Walraven photometry (bottom panel of Fig. 3). We note that CCs identified by the author as having known or suspected companions, or having other peculiarities, were not included in the figure. The agreement is once again good over the entire period range in common. We note that this approach is only based on photometric measurements. 

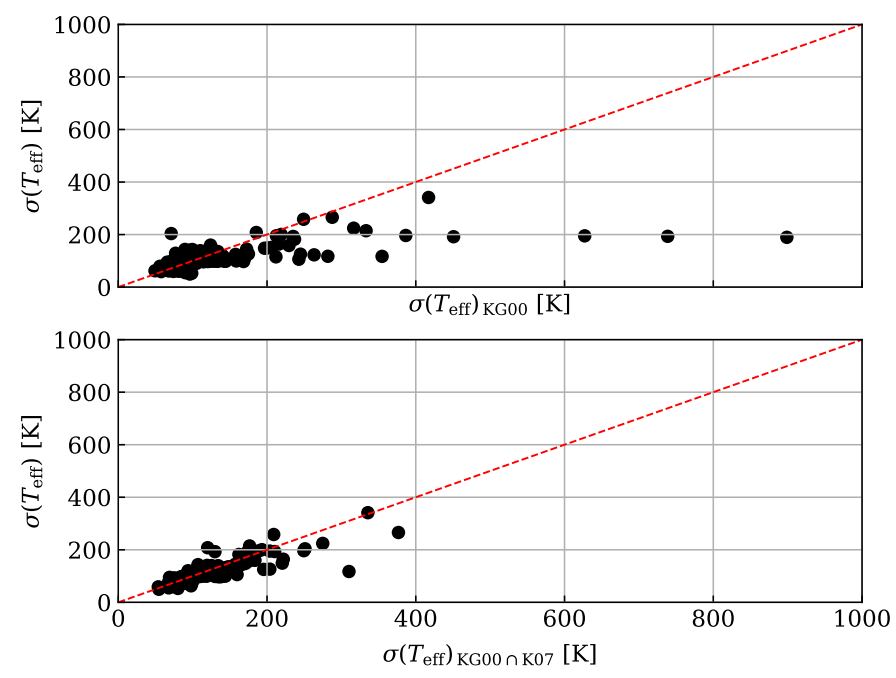

Fig. 2. Standard deviations of the effective temperature estimates for the 199 HARPS spectra. Top panel: entire set of 257 LDR calibrations used in the current investigation ( $\mathrm{KG} 00 \cap \mathrm{K} 07 \cap \mathrm{K} 18$ ) is compared with the 32 calibrations provided by KG00. Bottom panel: same as in top panel, but the comparison is between this study and the calibrations provided by $\mathrm{KG} 00 \cap \mathrm{K} 07$.

\subsection{Surface gravity and microturbulent velocity}

The surface gravity $(\log g)$ was derived through the ionization equilibrium between $\mathrm{Fe}_{\mathrm{I}}$ and $\mathrm{Fe}$ II lines and the microturbulent velocity $\left(v_{t}\right)$ was derived by minimizing the slope in the $[\mathrm{Fe} \mathrm{I} / \mathrm{H}]$ versus EW plot. This means that the $\log g$ value is changed until the $\mathrm{Fe}_{\mathrm{I}}$ and $\mathrm{Fe}$ II lines provide the same abundance, within the errors, while the $v_{t}$ value is changed until the dependence of the derived abundances on the EWs is removed. Indeed, weak and strong lines are supposed to provide the same elemental abundance. In the iterative procedure, the $T_{\text {eff }}$ values (derived as described in Sect. 4.2) are kept fixed, and the $\log g$ and $v_{t}$ values are changed until the aforementioned conditions are satisfied.

For the determination of these parameters, we used the MOOG LTE radiative code (Sneden 2002) applied to model atmospheres derived by interpolation in the grid of Castelli \& Kurucz (2004). We did not perform a specific test to constrain the difference when using different grids of atmosphere models. However, recent detailed results available in the literature (Heiter \& Eriksson 2006; Gustafsson et al. 2008) support a very good agreement in the spectral range $(\mathrm{F}-\mathrm{K})$ typical of classical Cepheids. The standard solar abundances adopted by the MOOG code (version of July 2014) come from Asplund et al. (2009). Although they have been recently revised by Grevesse et al. (2015) and by Scott et al. (2015a, b), we decided to use the abundances from Asplund et al. (2009) for consistency with our previous spectroscopic analyses.

Table 2 lists the weighted mean of the surface gravity and microturbulent velocity computed for the 14 calibrating Cepheids using the multiple values listed in Table 4. In that table, the uncertainties on the individual estimates of $\log g$ and $v_{t}$ are not listed, but they are expected to be on the order of $\sim 0.3$ dex and $\sim 0.5 \mathrm{~km} \mathrm{~s}^{-1}$, respectively (see Genovali et al. 2014). In Table 2, the uncertainty on the weighted mean is shown.

In the top panel of Fig. 4 we show the comparison among the current surface gravity amplitudes $(\Delta \log g)$ and similar estimates by Pel (1978) and by S11. As in Fig. 3, stars from Pel (1978) with known or suspected companions, or having other peculiarities, were not included. The three different datasets agree very well within the errors. We note that the two Cepheids with larger surface gravity amplitudes (UZ Sct and AV Sgr) are among those for which the phase coverage is not optimal. The bottom panel of the same figure shows the comparison between the current microturbulent velocity amplitudes and similar estimates provided by Luck \& Lambert (2011; hereinafter LL11) for Cepheids with multiple measurements. The two datasets agree very well once we take account of the fact that several targets in the LL11 sample only have a few spectra. The same applies for the targets for which we do not have an optimal phase coverage. The surface gravity and microturbulent velocity amplitudes will be discussed in more detail in a forthcoming paper (Urbaneja et al., in prep.) using an independent spectroscopic approach.

\subsection{Radial velocity estimates}

Radial velocity measurements were performed for the entire spectroscopic dataset, i.e., we also included the spectra for which the $\mathrm{S} / \mathrm{N}$ was not good enough for the spectroscopic analysis. The radial velocity curves as a function of the pulsation phase are shown in Fig. 5. Typical radial velocity errors are around $0.1 \mathrm{~km} \mathrm{~s}^{-1}$, and normally smaller than $0.5 \mathrm{~km} \mathrm{~s}^{-1}$, which is often smaller than the symbol size in the figure. The pulsation phase corresponding to each observed spectrum has been computed on the basis of the pulsation period and the photometric data ( $V$ band) available in the literature (Groenewegen 2008; Storm et al. 2011a). However, in most cases the time span between the photometric and spectroscopic observation is larger than 30 years. On such long timescales, Cepheids change their period owing to evolutionary effects, and by using an outdated period we would introduce a scatter in the folded curves. In order to avoid this effect, we combined the photometric and spectroscopic data to compute a more accurate period by using a generalized Lomb-Scargle algorithm. The new periods are listed in Table 1 , together with the zero-phase reference epoch $\left(T_{0}\right)$ corresponding to maximum light in the $V$ band.

To validate the radial velocity amplitudes estimated for the calibrating Cepheids, Fig. 6 shows the comparison between the current values and those provided by S11 for IRSB Galactic Cepheids. We note that only two stars (Y Oph and XX Sgr) are indicated with black crosses because the phase coverage is better in the RV plots. The two datasets agree very well over the entire period range and display the expected V-shape across the Hertzsprung progression $(\log P \sim 1.0)$. In this context, we would like to draw the attention to a small sample of Cepheids with periods ranging from $\log P \sim 1.1$ to $\log P \sim 1.6$ that, at fixed period, display radial velocity amplitudes that are on average a factor of two smaller than the bulk of Cepheids. One possible culprit could be the metallicity, since there is preliminary evidence that the amplitudes might decrease when moving into the more metal-poor regime (see Fig. 11 in Genovali et al. 2014). The quality and homogeneity of the spectra we are collecting will allow us, on a timescale of a few years, to provide more quantitative constraints on this working hypothesis.

\section{Iron abundance determinations}

The output file of the MOOG code provides the iron abundance for each one of the FeI and Fe II lines passed as input. The current estimates, when compared with similar estimates available in the literature, present several advantages: (i) Our sample has between 5 and more than 100 spectra per star. This is the reason why the current mean iron abundances 
B. Proxauf et al.: A new and homogeneous metallicity scale for Galactic classical Cepheids. I.

Table 2. Mean parameters derived for the calibrating Cepheids.

\begin{tabular}{lccccccc}
\hline \hline Name & $\left\langle T_{\text {eff }}\right\rangle \pm \sigma(\mathrm{K})$ & $\langle\log g\rangle \pm \sigma$ & $\left\langle v_{t}\right\rangle \pm \sigma\left(\mathrm{km} \mathrm{s}^{-1}\right)$ & {$[\mathrm{Fe} \mathrm{I} / \mathrm{H}] \pm \sigma$} & {$[\mathrm{Fe} \mathrm{II} / \mathrm{H}] \pm \sigma$} & {$[\mathrm{Fe} / \mathrm{H}] \pm \sigma(\mathrm{std})$} & $N_{\text {spec }}$ \\
\hline V340 Ara & $5293 \pm 42$ & $1.14 \pm 0.11$ & $4.77 \pm 0.19$ & $0.24 \pm 0.07$ & $0.22 \pm 0.05$ & $0.23 \pm 0.04(0.07)$ & 7 \\
$\eta$ Aql & $5479 \pm 39$ & $1.11 \pm 0.09$ & $3.43 \pm 0.15$ & $0.26 \pm 0.05$ & $0.23 \pm 0.03$ & $0.24 \pm 0.03(0.09)$ & 11 \\
S Cru & $6014 \pm 21$ & $1.64 \pm 0.08$ & $3.08 \pm 0.14$ & $0.09 \pm 0.03$ & $0.08 \pm 0.04$ & $0.09 \pm 0.03(0.04)$ & 13 \\
$\beta$ Dor & $5557 \pm 13$ & $1.35 \pm 0.04$ & $3.78 \pm 0.07$ & $-0.04 \pm 0.02$ & $-0.02 \pm 0.02$ & $-0.03 \pm 0.01(0.05)$ & 46 \\
$\zeta$ Gem & $5494 \pm 7$ & $1.12 \pm 0.03$ & $3.22 \pm 0.04$ & $0.15 \pm 0.01$ & $0.17 \pm 0.01$ & $0.16 \pm 0.01(0.05)$ & 128 \\
Y Oph & $5612 \pm 33$ & $0.99 \pm 0.11$ & $3.22 \pm 0.18$ & $0.06 \pm 0.05$ & $0.09 \pm 0.06$ & $0.08 \pm 0.04(0.05)$ & 8 \\
RS Pup & $5381 \pm 27$ & $0.84 \pm 0.08$ & $4.66 \pm 0.13$ & $0.13 \pm 0.04$ & $0.15 \pm 0.03$ & $0.14 \pm 0.02(0.07)$ & 14 \\
UZ Sct & $5038 \pm 36$ & $1.24 \pm 0.11$ & $4.86 \pm 0.18$ & $0.13 \pm 0.07$ & $0.07 \pm 0.09$ & $0.11 \pm 0.05(0.09)$ & 8 \\
AV Sgr & $5228 \pm 38$ & $1.18 \pm 0.11$ & $4.86 \pm 0.18$ & $0.25 \pm 0.07$ & $0.32 \pm 0.03$ & $0.31 \pm 0.02(0.08)$ & 8 \\
VY Sgr & $5340 \pm 35$ & $0.98 \pm 0.09$ & $4.59 \pm 0.16$ & $0.21 \pm 0.06$ & $0.27 \pm 0.04$ & $0.25 \pm 0.03(0.08)$ & 10 \\
XX Sgr & $5843 \pm 41$ & $1.30 \pm 0.13$ & $2.98 \pm 0.22$ & $0.09 \pm 0.06$ & $0.03 \pm 0.06$ & $0.06 \pm 0.04(0.02)$ & 5 \\
Y Sgr & $5924 \pm 26$ & $1.75 \pm 0.06$ & $4.11 \pm 0.11$ & $0.07 \pm 0.03$ & $-0.02 \pm 0.02$ & $0.00 \pm 0.01(0.06)$ & 22 \\
R TrA & $6039 \pm 25$ & $1.97 \pm 0.08$ & $4.01 \pm 0.13$ & $0.02 \pm 0.03$ & $-0.04 \pm 0.03$ & $-0.01 \pm 0.02(0.03)$ & 15 \\
RZ Vel & $5479 \pm 29$ & $1.23 \pm 0.09$ & $4.62 \pm 0.14$ & $0.09 \pm 0.04$ & $0.08 \pm 0.04$ & $0.08 \pm 0.03(0.06)$ & 12 \\
\hline
\end{tabular}

Notes. From left to right the columns give the name, effective temperature, surface gravity, microturbulent velocity, iron abundances, and number of spectra used to compute the mean values. These are the weighted mean and its uncertainty computed from the values in Table 4 . The standard deviation of the mean computed using individual abundances of both $\mathrm{Fe}_{\mathrm{I}}$ and $\mathrm{Fe}_{\mathrm{II}}$ is also shown.

Table 3. Excerpt from the list of new and old LDRs adopted for effective temperature estimates.

\begin{tabular}{|c|c|c|c|c|c|c|c|c|c|c|c|c|}
\hline$\lambda_{1}[\AA]$ & Ion & $\lambda_{2}[\AA]$ & Ion & $\Delta T_{\mathrm{eff}}[\mathrm{K}]$ & $a$ & $b$ & $c$ & $d$ & $e$ & $f$ & Function & Ref. \\
\hline 5348.30 & Cr I & 5554.89 & $\mathrm{Fe}_{\mathrm{I}}$ & $7200-7700$ & 8120 & -919.996 & $\ldots$ & $\cdots$ & $\cdots$ & & $a+b r$ & 2 \\
\hline 5348.30 & Cr I & 5565.71 & $\mathrm{Fe}_{\mathrm{I}}$ & $7200-7700$ & 7940 & -646.94 & $\ldots$ & $\ldots$ & $\ldots$ & $\ldots$ & $a+b r$ & 2 \\
\hline$\ldots$ & $\ldots$ & $\ldots$ & $\ldots$ & $\ldots$ & $\ldots$ & $\ldots$ & $\ldots$ & $\ldots$ & $\ldots$ & $\ldots$ & $\ldots$ & $\ldots$ \\
\hline 7912.87 & $\mathrm{Fe}_{\mathrm{I}}$ & 7710.37 & $\mathrm{Fe}_{\mathrm{I}}$ & $5300-6800$ & 7433.4687 & -3131.7646 & 1119.1743 & $\cdots$ & $\cdots$ & $\cdots$ & $a+b r+c r^{2}$ & 3 \\
\hline 8426.51 & Ti I & 7680.27 & $\mathrm{Si}$ I & $5300-6400$ & 6963.6582 & -2717.0084 & 967.28678 & $\ldots$ & $\ldots$ & $\ldots$ & $a+b r+c r^{2}$ & 3 \\
\hline
\end{tabular}

Notes. From left to right, the first four columns give the wavelength of the line pairs adopted for the LDR, while Col. 5 gives the range in effective temperature in which the individual LDRs were calibrated. Columns 6-11 list the coefficients of the analytical relation adopted for the calibration, while Col. 12 gives the analytical formula. The last column gives the reference for the calibration of the LDR. The complete table is available at the CDS.

References. ${ }^{(1)}$ Kovtyukh \& Gorlova (2000, KG00); ${ }^{(2)}$ Kovtyukh (2007, K07); ${ }^{(3)}$ K18: this investigation.

Table 4. Excerpt from the list of atmospheric parameters, Fe abundances, and radial velocities for the spectra of the 14 calibrating CCs.

\begin{tabular}{|c|c|c|c|c|c|c|c|c|c|c|c|}
\hline Name & Dataset & $\operatorname{MJD}(\mathrm{d})$ & $T_{\mathrm{eff}} \pm \sigma(\mathrm{K})$ & $\log g$ & $v_{t}\left(\mathrm{~km} \mathrm{~s}^{-1}\right)$ & $\mathrm{Fe}_{\mathrm{I}} \pm \sigma$ & $N_{\mathrm{Fe}_{\mathrm{I}}}$ & Fe II $\pm \sigma$ & \multicolumn{3}{|c|}{$N_{\mathrm{Fe}_{\text {II }}}[\mathrm{Fe} / \mathrm{H}] \pm \sigma R V \pm \sigma\left(\mathrm{km} \mathrm{s}^{-1}\right)$} \\
\hline V340 Ara & FEROS & 620.0609687 & $5921 \pm 154$ & 2.0 & 4.9 & $0.29 \pm 0.22$ & 85 & $0.05 \pm 0.13$ & 4 & $0.11 \pm 0.11$ & $-76.9 \pm 5.3$ \\
\hline V340 Ara & UVES & 708.0671613 & $5181 \pm 99$ & 0.9 & 4.3 & $0.27 \pm 0.20$ & 86 & $0.24 \pm 0.26$ & 7 & $0.26 \pm 0.16$ & $-95.69 \pm 0.03$ \\
\hline$\ldots$ & $\ldots$ & $\ldots$ & $\ldots$ & $\ldots$ & $\ldots$ & & $\ldots$ & $\ldots$ & $\ldots$ & & \\
\hline RZ Vel & FEROS & 280.0861358 & $5199 \pm 99$ & 1.2 & 4.9 & $-0.05 \pm 0.28$ & 93 & $0.08 \pm 0.17$ & 6 & $0.04 \pm 0.15$ & $49.1 \pm 2.3$ \\
\hline RZ Vel & HARPS & 606.1850289 & $5308 \pm 110$ & 1.6 & 4.9 & $0.18 \pm 0.20$ & 54 & $0.16 \pm 0.27$ & 5 & $0.18 \pm 0.16$ & $47.9 \pm 2.6$ \\
\hline
\end{tabular}

Notes. The first three columns give the name of the target, spectroscopic dataset, and Modified Julian Date at which the spectrum was collected. Columns 4-6 give the effective temperature and its standard deviation, surface gravity, and microturbulent velocity. Cols. 7-8 and 9-10 list both $\mathrm{Fe}_{\mathrm{I}}$ and $\mathrm{Fe}$ II abundances and their standard deviations together with the number of lines adopted for the measurements. Column 11 gives the weighted mean of $\mathrm{Fe}_{\mathrm{I}}$ and $\mathrm{Fe}$ II abundances (weighted by $1 / \sigma^{2}$ ) with its intrinsic error, while the last column gives the radial velocity measurement and the respective uncertainty. The complete table is available at the CDS.

have intrinsic errors smaller than 0.1 dex. Before the present work, there were only a few classical Cepheids for which multiple measurements were available (see, e.g., Luck \& Andrievsky 2004; Luck et al. 2008; Romaniello et al. 2008; Genovali et al. 2014); (ii) The current high-resolution spectra cover both the rising and decreasing branch. This means that they cover the pulsation phases during which Cepheids experience the largest variations in effective temperature, surface gravity, and microturbulent velocity; (iii) The current calibrating Cepheids roughly cover the period range typical of Galactic classical Cepheids, i.e., from $\sim 3$ to more than 40 days. This means that the current sample in the Bailey diagram (luminosity amplitude versus logarithmic period) covers both the low and large amplitude regime. Moreover, we are also sampling the region of Bump Cepheids. Classical Cepheids with periods ranging from $\sim 7$ to $\sim 10$ days display a well-defined bump either along the rising (shorter periods) or along the decreasing (longer periods) branch. This is the so-called Hertzsprung progression. The physical 
Table 5. Excerpt from the list of high-resolution spectra only adopted for radial velocity measurements.

\begin{tabular}{lccc}
\hline \hline Name & Dataset & MJD $(\mathrm{d})$ & $R V \pm \sigma\left(\mathrm{km} \mathrm{s}^{-1}\right)$ \\
\hline V340 Ara & FEROS & 53520.2213553 & $-99.7 \pm 7.5$ \\
V340 Ara & FEROS & 53521.2126174 & $-96.7 \pm 5.8$ \\
$\ldots$ & $\ldots$ & $\ldots$ & $\ldots$ \\
VY Sgr & FEROS & 53620.1598186 & $3.1 \pm 0.9$ \\
Y Sgr & HARPS & 56553.1423824 & $-13.4 \pm 1.0$ \\
\hline
\end{tabular}

Notes. From left to right the columns give the name of the target, spectroscopic dataset, modified Julian Date, and radial velocity measurements together with their standard deviations. The complete table is available at the CDS.

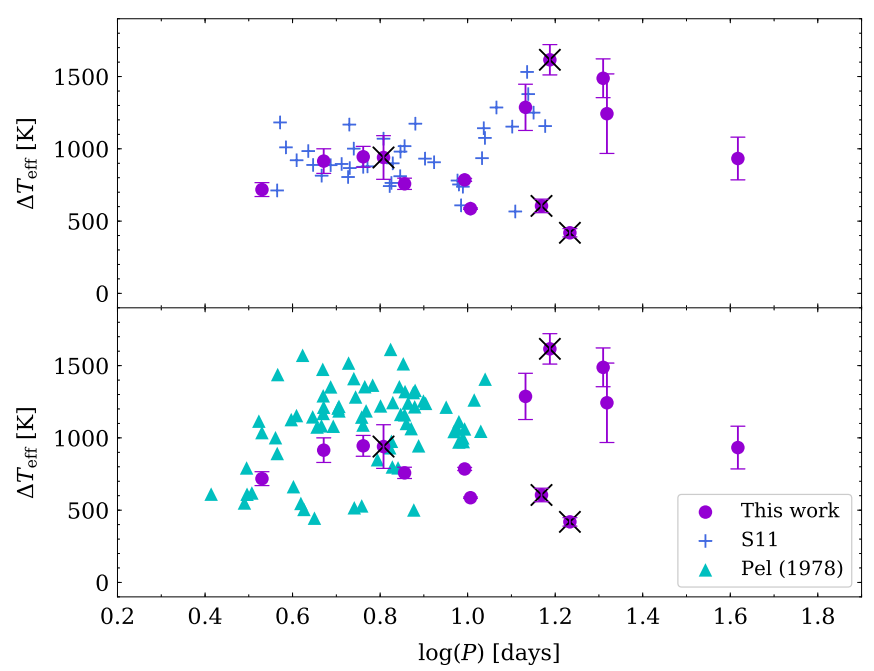

Fig. 3. Effective temperature amplitude $\left(\Delta T_{\text {eff }}=T_{\text {eff }}{ }^{\max }-T_{\text {eff }}{ }^{\min }\right)$ as a function of the logarithmic period. The black crosses indicate stars for which the available spectra do not properly cover the maximum and minimum of the pulsation cycle.

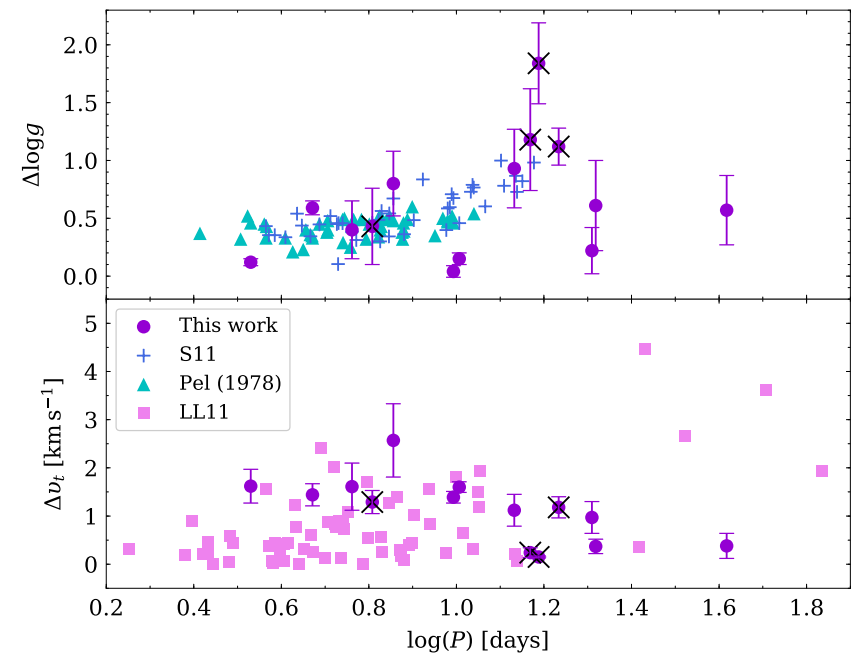

Fig. 4. Same as in Fig. 3, but showing the surface gravity amplitude (top panel) and the microturbulent velocity amplitude (bottom panel).

mechanisms driving the occurrence of this phenomenon are not fully understood yet, but there is mounting evidence that it is driven by nonlinear phenomena (shocks) across the entire envelope (Bono et al. 2000b).
The individual $\mathrm{Fe} \mathrm{I}$ and $\mathrm{Fe}$ II abundances and their uncertainties, together with the number of lines used are listed in Table 4. The number of lines measured for $\mathrm{Fe}_{\mathrm{I}}$ lines ranges from a few tens to more than 100, while for Fe II it ranges from a few to almost two dozen. These are smaller than the total number of lines available (570 for $\mathrm{Fe}_{\mathrm{I}}$ and 45 for $\mathrm{Fe}$ II) because the number of lines measured in each spectrum is limited, e.g., by the wavelength range covered by the instruments, the intensity of the lines for a given spectral type, their quality, and the level of blending. Moreover, for each spectrum, we performed a cleaning to remove lines that systematically provided abundances that are too high or too low (outside $2 \sigma$ ) when compared with the average abundance.

The mean Fe I and Fe II abundances together with the mean intrinsic parameters are listed in Table 2. A glance at the data given in this table indicates that mean abundances based either on neutral or on ionized iron have similar errors.

The comparison between current mean iron abundances and similar estimates available in the literature (see Table 1) indicates that they agree very well within the errors. The iron abundances and their errors listed in Table 1 come from Genovali et al. (2014), in which they derived spectroscopic abundances for the entire sample of CCs based on high-resolution optical spectra. For the measurements for which the original authors did not provide an estimate of the error, Genovali et al. assumed a typical error of 0.1 dex. This means that the difference between the current mean iron abundances and similar estimates available in the literature is, on average, smaller than $1 \sigma$. There is only one exception, $\zeta \mathrm{Gem}$, for which the difference is on the order of $3 \sigma$. The reason for this difference is not clear. $\zeta$ Gem is the object with the highest precision, since we analyzed 128 spectra and they cover the entire pulsation cycle. Moreover, the variation of the physical parameters (see Fig. 8) is smooth during both rising and decreasing branch, and both $\mathrm{Fe}_{\mathrm{I}}$ and $\mathrm{Fe}$ II estimates display minimal variations along the entire pulsation cycle of this object (see Fig. 9). Nevertheless, we should notice that $\zeta$ Gem is a peculiar Cepheid that has been investigated by Szabados (1983) and classified as a variable star having secular period changes. Indeed, its radial velocity clearly changes with the epoch, as can be seen in Fig. 5, not just owing to a velocity offset but real phase shifts seem to be observed, possibly caused by an unseen companion.

To further quantify the difference between the current iron abundances and similar abundances available in the literature, Fig. 7 displays the comparison with the metallicity gradient of Galactic Cepheids provided by Genovali et al. (2014). Data plotted in this figure show some interesting results: $(a)$ The current sample follows very well the global metallicity gradient and the new homogeneous mean abundances display a smaller dispersion compared with the literature values ( 0.10 vs. 0.12 dex). (b) The new mean abundances of the four innermost disk objects (V340 Ara, UZ Sct, VY Sgr, AV Sgr) are systematically more metal poor than literature estimates. This means that the determination of the metallicity gradient in the transition zone between the inner disk and the Bulge (Bono et al. 2013; Genovali et al. 2014) will strongly benefit from more homogeneous and accurate mean iron abundances (Inno et al., in prep.).

\section{Phase dependence}

In the determination of the effective temperature, high standard deviations are not automatically linked to problems in the linedepth measurements. Spectra of stars in the rising phase of their 
B. Proxauf et al.: A new and homogeneous metallicity scale for Galactic classical Cepheids. I.
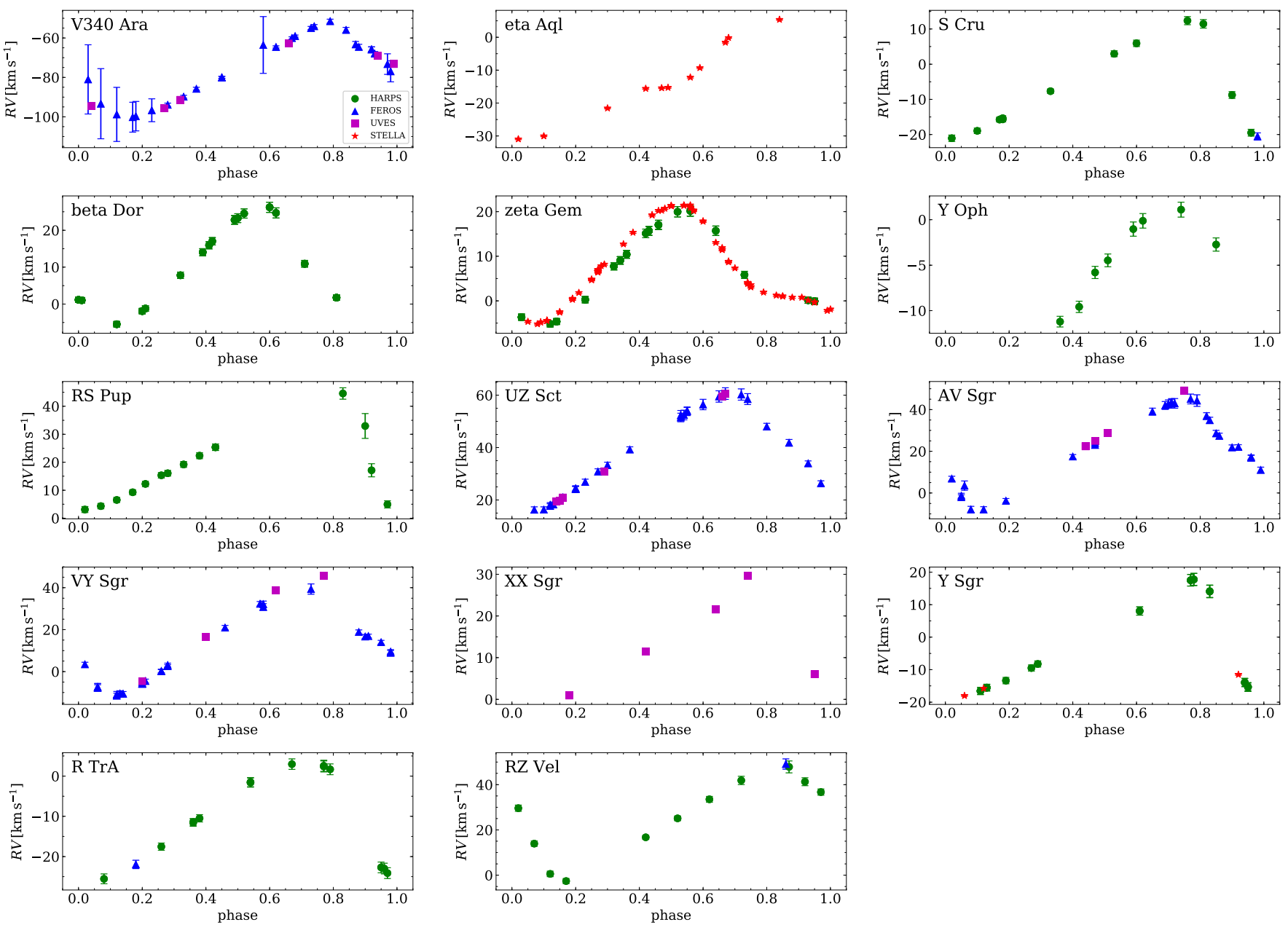

Fig. 5. Radial velocities as a function of the pulsation phase. Measurements based on different spectrographs are indicated with different colors and symbols. The error bars in some cases are smaller than the symbol size.

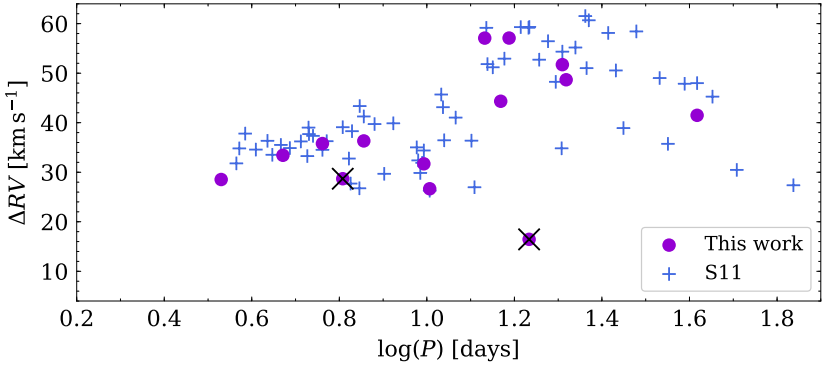

Fig. 6. Same as in Fig. 3, but showing the radial velocity amplitude.

pulsation cycle (i.e., when their effective temperature is increasing) present themselves with intrinsically higher dispersions due to the variable nature of the star; the spectra are thus fixed by the physical structure. This could be checked only for the spectra with multiple measurements.

The dependence of the effective temperature on the pulsation phase is shown in Fig. 8. The same figure also shows the variation of the surface gravity and microturbulent velocity with the pulsation phase, but the dependence is very weak given the uncertainties. It is worth mentioning that the microturbulent velocity peaks around the pulsation phases in which the Cepheid attains its lowest effective temperatures and soon after, i.e., the phases between $\sim 0.5$ and $\sim 0.7 / 0.8$ (see in Fig. 8 : S Cru, $\beta$ Dor,

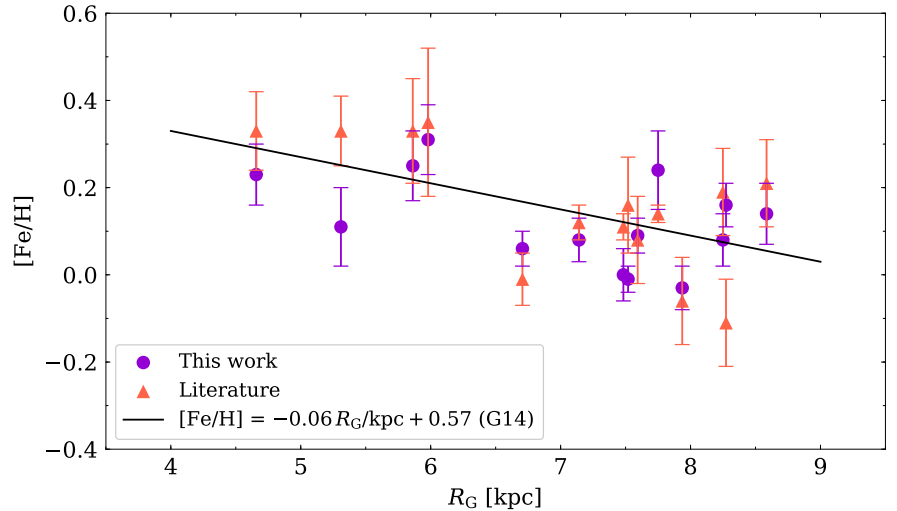

Fig. 7. Iron abundances as a function of Galactocentric distances for the calibrating Cepheids. Values derived in the present work (Table 2) are compared with those from the literature (Table 1). The error bars on our metallicity estimates are the largest value between the uncertainty on the weighted mean and the standard deviation. The metallicity gradient derived by Genovali et al. (2014) is also shown.

$\zeta$ Gem, R TrA). This evidence supports earlier findings by Luck \& Andrievsky (2004), Kovtyukh et al. (2005), Andrievsky et al. (2005), and Luck et al. (2008) suggesting that the microturbulent velocity peaks around phases $0.6-0.8$. A more quantitative comparison is hampered by the difference in the targets and phase 

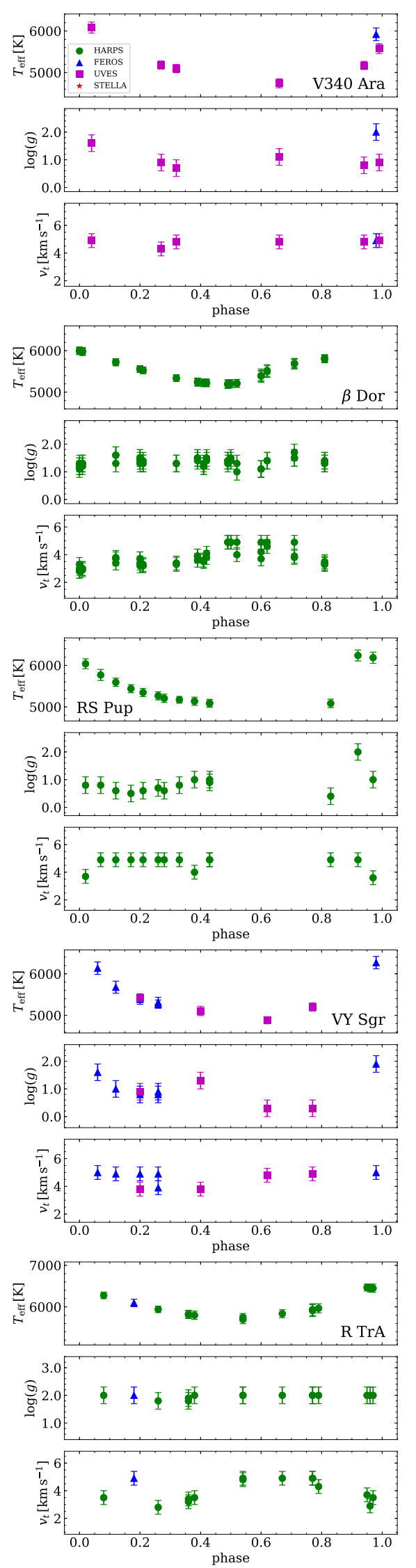
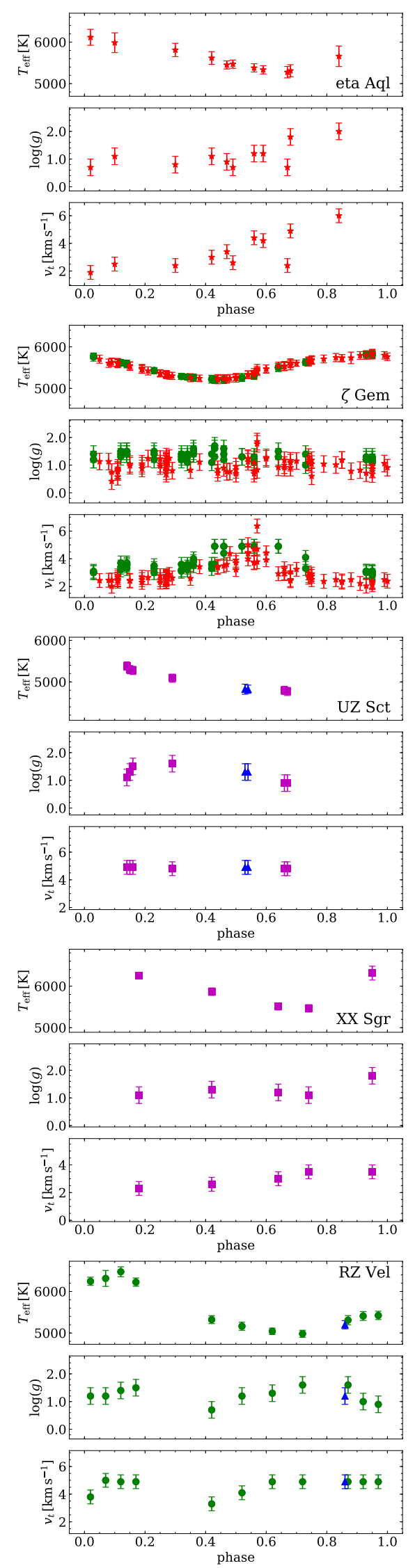
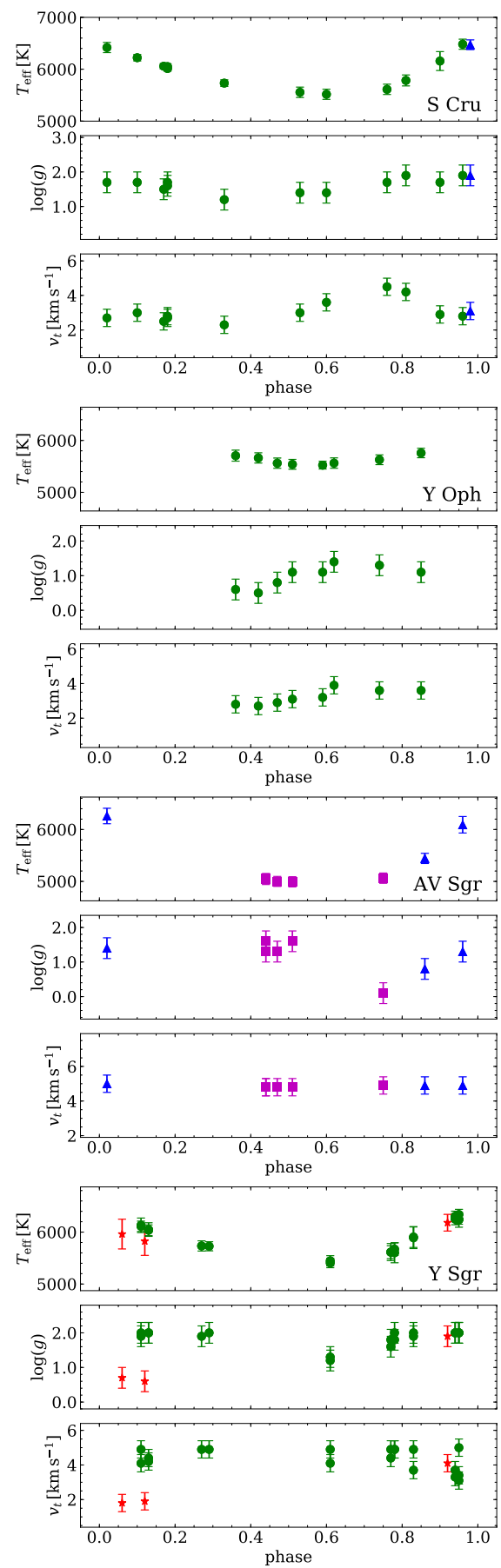

Fig. 8. Atmospheric parameters as a function of the pulsation phase. Measurements from different spectrographs are indicated with different colors and symbols. To help with the comparison, the panels are plotted with same $y$-axis range: $2000 \mathrm{~K}$ for $T_{\text {eff }}, 3$ dex for $\log g$, and $6 \mathrm{~km} \mathrm{~s}{ }^{-1}$ for $v_{t}$. 
B. Proxauf et al.: A new and homogeneous metallicity scale for Galactic classical Cepheids. I.
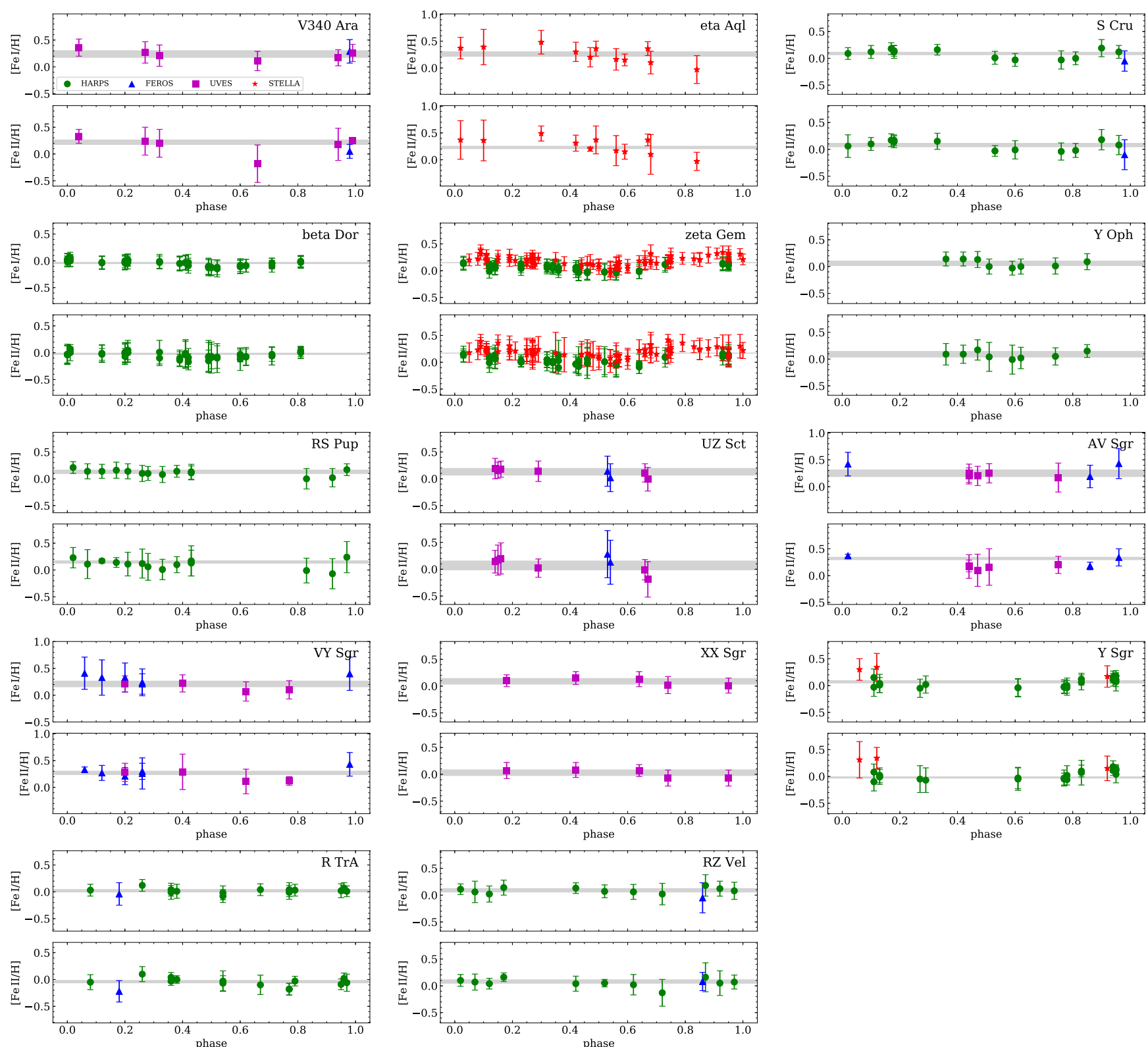

Fig. 9. Abundances from $\mathrm{Fe}_{\mathrm{I}}$ and $\mathrm{Fe}$ II lines as a function of the pulsation phase. The color coding of the various points is the same as in Fig. 8 . To help with the comparison, the panels are plotted with same $y$-axis range: 1.5 dex for both $\mathrm{Fe}_{\mathrm{I}}$ and $\mathrm{Fe}$ II panels. The light gray shaded regions indicate the $\pm 1 \sigma$ uncertainty around the weighted mean (from columns 5 and 6 of Table 2).

coverage. We still lack detailed empirical constraints on the variation of the microturbulent velocity as a function of the pulsation period, and in particular, across the Hertzsprung progression. Homogeneous spectra covering the entire pulsation cycle and a broad period range are highly desired. The same outcome applies to the different approaches suggested to trace the variation of convective motions (Gillet et al. 1999).

Finally, we note that data plotted in Fig. 9 clearly show that $\mathrm{Fe}$ I and Fe II abundances agree very well within the errors. Moreover and even more importantly, they are independent of the pulsation phase.

\section{Summary and final remarks}

The quoted results bring forward a few relevant issues that are worthy to discuss in more detail.

High-resolution, high $\mathrm{S} / \mathrm{N}$ optical spectra of variable stars allow us to provide precise estimates of both physical parameters and abundances along the pulsation cycle. This means that spectra collected at random phases can provide solid estimates of Cepheid elemental abundances. This argument applies for relative measurements. Solid constraints on the possible occurrence of systematics in the zero-point of physical parameters and in elemental abundances do require independent spectroscopic approach based either on spectral synthesis and/or on an NLTE analysis.

The observational scenario concerning the LDRs in the NIR regime is lagging compared with the optical scenario, after the seminal investigations by Sasselov et al. (1989) and Sasselov \& Lester $(1990 a, b)$ have been hampered by the lack of efficient echelle NIR spectrographs. Fortunately, recent investigations are paving the way for an extension of the LDR into the NIR regime. Fukue et al. (2015) collected $H$-band spectra with a Subaru high-resolution camera and spectrograph (Kobayashi et al. 2000) for several G and K-type giants and supergiants. Interestingly enough, these authors found that they can 
provide effective temperatures with an accuracy on the order of $60 \mathrm{~K}$ in spite of the limited range in wavelengths $(1.4-1.8 \mu \mathrm{m})$ covered by their spectra and the limited number of pairs (nine) they used. Taniguchi et al. (2018), using high-resolution spectra collected with WINERED (Ikeda et al. 2016) in the Y and $J$ bands $(0.9-1.35 \mu \mathrm{m})$ for ten early $\mathrm{G}$ and M-type giants, found $81 \mathrm{LDR}-T_{\text {eff }}$ relations, achieving a precision of $10 \mathrm{~K}$ in the best cases. These findings appear as a very promising opportunity for future developments of NIR spectrographs, such as WINERED (see also D'Orazi et al. 2018), CRIRES+ (Follert et al. 2014), and GIANO (Origlia et al. 2016).

The new calibrations of the LDRs presented in this paper, together with similar calibrations available in the literature cover, for the first time, the same range in effective temperature covered by CCs along their pulsation cycles. However, the range in metallicity covered by the current Cepheids is roughly half a dex around solar metallicity. New extensions into the more metalpoor and metal-rich regime are highly encouraged.

The estimate of the surface gravity using the ionization equilibrium between $\mathrm{Fe}_{\mathrm{I}}$ and $\mathrm{Fe}$ II lines is very robust, but new approaches are required for metal-poor objects and/or for NIR spectra in which the number of metallic lines is limited. The next Gaia release (DR2), by including accurate estimates of geometrical distances, photometry, and spectroscopy, will constrain the variation of surface gravity for static and variable stars. This is a unique opportunity to constrain possible systematics.

The anti-correlation between microturbulent velocity and effective temperature is very interesting. Further investigations to derive analytical relations can pave the way to a better understanding of the physical mechanisms (convective transport, nonlinear phenomena) driving the efficiency of microturbulent velocity along the pulsation cycle. One-dimensional non-LTE static atmosphere models and 3D dynamical atmosphere models (Chiavassa et al. 2018) would be highly desirable to investigate the physical phenomena affecting line formation and abundances in variable stars.

The current long-term variability surveys are discovering hundreds, or even thousands, of classical Cepheids along the obscured regions of the Galactic plane (Udalski et al. 2018, priv. comm.). The new identifications together with fiber multiobject (4MOST, MOONS, APOGEE-South, WEAVE) and slit NIR spectrographs (CRIRES+, WINERED, GIANO, CARMENES) will provide a unique opportunity to investigate the chemical enrichment of young stellar populations across the Galactic thin disk.

Acknowledgements. L. Inno acknowledges the Sonderforschungsbereich SFB 881 "The Milky Way System" (subproject A3) of the German Research Foundation (DFG).

\section{References}

Anderson, R. I., Saio, H., Ekström, S., Georgy, C., \& Meynet, G. 2016, A\&A, 591, A8

Andrievsky, S. M., Bersier, D., Kovtyukh, V. V., et al. 2002a, A\&A, 384, 140

Andrievsky, S. M., Kovtyukh, V. V., Luck, R. E., et al. 2002b, A\&A, 381, 32 Andrievsky, S. M., Luck, R. E., \& Kovtyukh, V. V. 2005, AJ, 130, 1880

Asplund, M., Grevesse, N., Sauval, A. J., \& Scott, P. 2009, ARA\&A, 47, 481

Baade, W. 1958, Ricerche Astronomiche, 5, 165

Bono, G., Caputo, F., Castellani, V., \& Marconi, M. 1999a, ApJ, 512, 711

Bono, G., Marconi, M., \& Stellingwerf, R. F. 1999b, ApJS, 122, 167

Bono, G., Castellani, V., \& Marconi, M. 2000a, ApJ, 529, 293

Bono, G., Marconi, M., \& Stellingwerf, R. F. 2000b, A\&A, 360, 245

Bono, G., Caputo, F., Marconi, M., \& Musella, I. 2010, ApJ, 715, 277

Bono, G., Matsunaga, N., Inno, L., Lagioia, E. P., \& Genovali, K. 2013

Cosmic Rays in Star-Forming Environments, eds. D.F. Torres, \& O. Reimer,

Astrophys. Space Sci. Proc., 34, 115
Castelli, F., \& Kurucz, R. L. 2004, ArXiv e-prints [ArXiv: astro-ph/0405087] Chiavassa, A., Casagrande, L., Collet, R., et al. 2018, A\&A, 611, A11

da Silva, R., Lemasle, B., Bono, G., et al. 2016, A\&A, 586, A125

Dekker, H., D’Odorico, S., Kaufer, A., Delabre, B., \& Kotzlowski, H. 2000, in Optical and IR Telescope Instrumentation and Detectors, eds. M. Iye, \& F. Moorwood, Proc. SPIE, 4008, 534

D’Orazi, V., Magurno, D., Bono, G., et al. 2018, ApJ, 855, L9

Evans, N. R. 1992, ApJ, 384, 220

Feast, M. W., Laney, C. D., Kinman, T. D., van Leeuwen, F., \& Whitelock, P. A. 2008, MNRAS, 386, 2115

Fiorentino, G., Marconi, M., Musella, I., \& Caputo, F. 2007, A\&A, 476, 863

Follert, R., Dorn, R.J., Oliva, E., et al. 2014, in Ground-based and Airborne Instrumentation for Astronomy, Proc. SPIE, V, 9147, 914719

François, P., Schuez, O., Conn, B., et al. 2006, FEROS-II User Manual, https://www.eso.org/sci/facilities/lasilla/instruments/ feros/doc/manual/P78/FEROSII-UserManual-78.0.pdf

Freedman, W. L., \& Madore, B. F. 2010, ARA\&A, 48, 673

Fukue, K., Matsunaga, N., Yamamoto, R., et al. 2015, ApJ, 812, 64

Gallenne, A., Kervella, P., Mérand, A., et al. 2014, A\&A, 567, A60

Genovali, K., Lemasle, B., Bono, G., et al. 2013, A\&A, 554, A132

Genovali, K., Lemasle, B., Bono, G., et al. 2014, A\&A, 566, A37

Genovali, K., Lemasle, B., da Silva, R., et al. 2015, A\&A, 580, A17

Gieren, W., Górski, M., Pietrzyński, G., et al. 2013, ApJ, 773, 69

Gillet, D., Fokin, A. B., Breitfellner, M. G., Mazauric, S., \& Nicolas, A. 1999, A\&A, 344, 935

Gilmore, G., Randich, S., Asplund, M., et al. 2012, The Messenger, 147, 25

Gray, D.F. 2005, The Observation and Analysis of Stellar Photospheres (Cambridge, UK: Cambridge University Press)

Grevesse, N., Scott, P., Asplund, M., \& Sauval, A. J. 2015, A\&A, 573, A27

Groenewegen, M. A. T. 2008, A\&A, 488, 25

Gustafsson, B., Edvardsson, B., Eriksson, K., et al. 2008, A\&A, 486, 951

Heiter, U., \& Eriksson, K. 2006, A\&A, 452, 1039

Hoffmann, S. L., Macri, L. M., Riess, A. G., et al. 2016, ApJ, 830, 10

Ikeda, Y., Kobayashi, N., Kondo, S., et al. 2016 in Ground-based and Airborne Instrumentation for Astronomy VI, Proc. SPIE, 9908, $99085 Z$

Kaufer, A., Stahl, O., Tubbesing, S., et al. 1999, The Messenger, 95, 8

Kervella, P., Bersier, D., Mourard, D., et al. 2004, A\&A, 428, 587

Kobayashi, N., Tokunaga, A. T., Terada, H., et al. 2000, in Optical and IR Telescope Instrumentation and Detectors, eds. M. Iye, \& A.F. Moorwood, Proc. SPIE, 4008, 1056

Kovtyukh, V. V. 2007, MNRAS, 378, 617

Kovtyukh, V. V., \& Andrievsky, S. M. 1999, A\&A, 350, L55

Kovtyukh, V. V., \& Gorlova, N. I. 2000, A\&A, 358, 587

Kovtyukh, V. V., Andrievsky, S. M., Luck, R. E., \& Gorlova, N. I. 2003a, A\&A, 401, 661

Kovtyukh, V. V., Soubiran, C., Belik, S. I., \& Gorlova, N. I. 2003b, A\&A, 411, 559

Kovtyukh, V. V., Andrievsky, S. M., Belik, S. I., \& Luck, R. E. 2005, AJ, 129, 433

Kovtyukh, V., Lemasle, B., Chekhonadskikh, F., et al. 2016, MNRAS, 460, 2077 Kraft, R. P. 1956, PASP, 68, 137

Kraft, R. P. 1957, ApJ, 125, 336

Krockenberger, M., Sasselov, D., Noyes, R., et al. 1998, in Cool Stars, Stellar Systems, and the Sun, eds. R. A. Donahue, \& J. A. Bookbinder, ASP Conf. Ser., 154, 791

Kurucz, R. L., Furenlid, I., Brault, J., \& Testerman, L. 1984, Solar flux atlas from 296 to $1300 \mathrm{~nm}$ (Sunspot, NM: National Solar Observatory)

Lemasle, B., François, P., Bono, G., et al. 2007, A\&A, 467, 283

Lemasle, B., François, P., Piersimoni, A., et al. 2008, A\&A, 490, 613

Lemasle, B., François, P., Genovali, K., et al. 2013, A\&A, 558, A31

Lemasle, B., Groenewegen, M., Grebel, E., et al. 2017, A\&A, 608, A85

Li Causi, G., Antoniucci, S., Bono, G., et al. 2013, A\&A, 549, A64

Luck, R. E., \& Andrievsky, S. M. 2004, AJ, 128, 343

Luck, R. E., \& Lambert, D.L. 2011, AJ, 142, 136

Luck, R. E., Moffett, T. J., Barnes, T.G., \& Gieren, W.P. 1998, AJ, 115, 605

Luck, R. E., Andrievsky, S. M., Fokin, A., \& Kovtyukh, V. V. 2008, AJ, 136, 98

Luck, R. E., Andrievsky, S. M., Kovtyukh, V. V., Gieren, W., \& Graczyk, D. 2011, AJ, 142, 51

Macri, L. M., Ngeow, C. C., Kanbur, S. M., Mahzooni, S., \& Smitka, M. T. 2015, AJ, 149, 117

Marconi, M., Musella, I., \& Fiorentino, G. 2005, ApJ, 632, 590

Mathias, P., Gillet, D., Fokin, A. B., et al. 2006, A\&A, 457, 575

Mayor, M., Pepe, F., Queloz, D., et al. 2003, The Messenger, 114, 20

Mérand, A., Kervella, P., Breitfelder, J., et al. 2015, A\&A, 584, A80

Nardetto, N., Gieren, W., Kervella, P., et al. 2009, A\&A, 502, 951

Origlia, L., Oliva, E., Sanna, N., et al. 2016, A\&A, 585, A14

Pel, J. W. 1978, A\&A, 62, 75

Pietrzyński, G., Graczyk, D., Gieren, W., et al. 2013, Nature, 495, 76 
B. Proxauf et al.: A new and homogeneous metallicity scale for Galactic classical Cepheids. I.

Preston, G. W. 1964, ARA\&A, 2, 23

Preston, G. W., Smak, J., \& Paczynski, B. 1965, ApJS, 12, 99

Randich, S., Gilmore, G., \& Gaia-ESO Consortium 2013, The Messenger, 154, 47

Riess, A. G., Macri, L. M., Hoffmann, S. L., et al. 2016, ApJ, 826, 56

Romaniello, M., Primas, F., Mottini, M., et al. 2008, A\&A, 488, 731

Ryabchikova, T., Piskunov, N., Kurucz, R. L., et al. 2015, Phys. Scr., 90, 054005

Sasselov, D. D., \& Lester, J. B. 1990a, ApJ, 360, 227

Sasselov, D. D., \& Lester, J. B. 1990b, ApJ, 362, 333

Sasselov, D. D., Fieldus, M. S., \& Lester, J. B. 1989, ApJ, 337, L29

Scott, P., Asplund, M., Grevesse, N., Bergemann, M., \& Sauval, A.J. 2015a, A\&A, 573, A26

Scott, P., Grevesse, N., Asplund, M., et al. 2015b, A\&A, 573, A25

Sneden, C. 2002, The MOOG code, https://www. as. utexas.edu/ chris/ moog.html

Soszyński, I., Udalski, A., Szymański, M. K., et al., 2017, Acta Astron., 67, 103

Sousa, S. G., Santos, N. C., Israelian, G., Mayor, M., \& Monteiro, M. J. P. F. G. 2007, A\&A, 469, 783

Sousa, S. G., Santos, N. C., Adibekyan, V., Delgado-Mena, E., \& Israelian, G. 2015, A\&A, 577, A67

Storm, J., Gieren, W., Fouqué, P., et al. 2011a, A\&A, 534, A94

Storm, J., Gieren, W., Fouqué, P., et al. 2011b, A\&A, 534, A95

Strassmeier, K. G., Granzer, T., Weber, M., et al. 2004, Astron. Nachr., 325, 527

Strassmeier, K. G., Granzer, T., Weber, M., et al. 2010, Adv. Astron., 2010, 970306

Struve, O. 1944, Observatory, 65, 257

Szabados, L. 1983, Ap\&SS, 96, 185

Szabados, L. 1990, MNRAS, 242, 285

Szabados, L. 2003, Inform. Bull. Variable Stars, 5394

Taniguchi, D., Matsunaga, N., Kobayashi, N., et al. 2018, MNRAS, 473, 4993

Vasilyev, V., Ludwig, H.G., Freytag, B., Lemasle, B., \& Marconi, M. 2017, A\&A, 606, A140

Vasilyev, V., Ludwig, H.G., Freytag, B., Lemasle, B., \& Marconi, M. 2018, A\&A, 611, A19

Wallerstein, G. 1972, PASP, 84, 656

Wallerstein, G. 1979, PASP, 91, 772

Wallerstein, G., Albright, M. B., \& Ritchey, A. M. 2015, PASP, 127, 503

Weber, M., Granzer, T., \& Strassmeier, K.G. 2012, in Software and Cyberinfrastructure for Astronomy II, Proc. SPIE, 8451, 84510K

1 Dipartimento di Fisica, Università degli Studi di Roma Tor Vergata, Via della Ricerca Scientifica 1, 00133 Rome, Italy e-mail: giuseppe.bono@roma2.infn.it

2 INAF - Osservatorio Astronomico di Roma, Via Frascati 33, 00078 Monte Porzio Catone, Rome, Italy
3 Agenzia Spaziale Italiana, Via del Politecnico snc, 00133 Rome, Italy e-mail: ronaldo.dasilva@ssdc.asi.it

4 Astronomical Observatory, Odessa National University, Shevchenko Park, 65014 Odessa, Ukraine

5 Isaac Newton Institute of Chile, Odessa Branch, Shevchenko Park, 65014 Odessa, Ukraine

6 Max-Planck Institute for Astronomy, 69117 Heidelberg, Germany

7 Astronomisches Rechen-Institut, Zentrum für Astronomie der Universität Heidelberg, Mönchhofstr. 12-14, 69120 Heidelberg, Germany

${ }^{8}$ Institute for Astro- and Particle Physics, University of Innsbruck, Technikerstr. 25/8, 6020 Innsbruck, Austria

${ }^{9}$ Leibniz-Institut für Astrophysik Potsdam, An der Sternwarte 16, 14482 Potsdam, Germany

${ }^{10}$ INAF - Osservatorio Astronomico di Teramo, Via Mentore Maggini snc, 64100 Teramo, Italy

11 INAF - Osservatorio Astronomico di Padova, Vicolo dell'Osservatorio 5, 35122 Padova, Italy

12 Monash Centre for Astrophysics, School of Physics and Astronomy, Monash University, Melbourne VIC 3800, Australia

13 INAF - Osservatorio Astronomico di Bologna, Via Ranzani 1, 40127 Bologna, Italy

14 GEPI, Observatoire de Paris, CNRS, Université Paris Diderot, Place Jules Janssen, 92190 Meudon, France

15 UPJV, Université de Picardie Jules Verne, 33 Rue St. Leu, 80080 Amiens, France

16 Department of Physics and Astronomy, N283 ESC, Brigham Young University, Provo, UT 84601, USA

17 South African Astronomical Observatory, PO Box 9, Observatory 7935, South Africa

18 Institute for Astronomy, University of Hawaii, 2680 Woodlawn Drive, Honolulu, HI 96822, USA

19 Max-Planck-Institute for Astrophysics, Karl-Schwarzschild-Str.1, 85741 Garching, Germany

20 University Observatory Munich, Scheinerstr. 1, 81679 Munich, Germany

21 Department of Astronomy, School of Science, The University of Tokyo, 7-3-1 Hongo, Bunkyo-ku, Tokyo 113-0033, Japan

22 INAF - Osservatorio Astronomico di Trieste, Via G. B. Tiepolo 11, 34143 Trieste, Italy

23 European Southern Observatory, Karl-Schwarzschild-Str. 2, 85748 Garching bei München, Germany

${ }^{24}$ Laboratoire Lagrange, CNRS/UMR 7293, Observatoire de la Côte d'Azur, Bd de l'Observatoire, CS 34229, 06304 Nice, France 